\title{
Morphological Variations of Chondrocytes in Bovine Articular Cartilage Using Confocal Laser Scanning Microscopy
}

\author{
E. ÇIÇEK* AND A. ARIKANOĞLU \\ Faculty of Science and Art, Mehmet Akif Ersoy University, Burdur, Turkey
}

\begin{abstract}
Articular cartilage is a highly organized avascular tissue, a matrix consisting of a three-dimensional meshwork of type II collagen fibrils and proteoglycans. To understand the effects of the alteration in chondrocyte shape in response to physiological load in local mechanical properties and, thus, to contribute to experimental load studies, we studied variation of the natural load induced deformations in chondrocytes among five different regions from bovine articular cartilage samples using confocal laser scanning microscopy imaging methods. The morphological differences of the chondrocyte shape, orientation and circularity in articular cartilage were measured. The chondrocytes were observed to be smoother in more loaded regions to the center of the cartilage.
\end{abstract}

DOI: $10.12693 /$ APhysPolA.125.898

PACS: 87.85.G-

\section{Introduction}

Articular cartilage, a highly organized smooth tissue covering the ends of the bones in articular mammalian joints is important for biomechanical mobility, protecting joint bones from high contact stresses and shearing forces $[1,2]$. Articular cartilage lesions are usually believed to develop severe forms of osteoarthritis [3-5].

Mechanical loading is an important factor in cartilage homeostasis. Articular cartilage tissue performs the essential biomechanical functions of load support enabling minimal damage throughout the lifetime [6]. Chondrocytes are sparsely distributed cells found in articular cartilage [7]. They react to mechanical strain and shear loading which influence the expression of superficial zone proteins $[8,9]$.

The load carriage stiffness of cartilage is provided by the mechanical effectiveness under compression. The matrix exudes water and exchanges small ions with its environment during deformation $[1,2]$.

In previous studies, the regional variations in human hip joint cartilage were studied with magnetic resonance both healthy and osteoarthritis groups [10, 11]. Topographical variations in the polarization sensitivity of bovine articular cartilage have been studied using polarization-sensitive optical coherence tomography and polarized light microscopy [12]. In experimental articular cartilage studies on load carriage in general, healthy cartilage samples are used, and natural deformations of the cellular shape and density due to physiological load have not been studied so far in different regions of the tibial cartilage using confocal microscope imaging methods. Thus the effects of deformations related to aging and load cannot be evaluated in vivo. We used confocal microscopy to understand the influence of topographical

*corresponding author; e-mail: ekrcicek@gmail.com variations in chondrocyte shapes and angles of bovine articular cartilage.

\section{Experimental}

Mature bovine stifle joints with intact capsules were obtained after slaughter from a local abattoir employing sterile techniques. Osteochondral explants $(\approx 540 \times$ $540 \mu \mathrm{m}^{2}$ width) were harvested from the lateral tibial plateau and transported in sterile sample tubes with HANKS buffered salt solution (supplemented with penicillin/streptomycin and amphotericin B), maintaining in vivo joint osmolarity. During the whole study to maintain tissue viability, the explants were allowed to equilibrate in phenol red-free culture media in standard low oxygen conditions $\left(5 \% \mathrm{O}_{2}, 5 \% \mathrm{CO}_{2}\right)$. This medium has $45 \%$ Dulbecco's modified essential medium, $45 \%$ Ham's F12, 10\% fetal bovine serum [13, 14]. The explants were harvested from five different regions of the lateral tibial plateau (Fig. 1).

The explants were placed for $30 \mathrm{~min}$ in media containing $5 \mu \mathrm{M}$ of the superoxide probe dihydroethidium (DHE; Invitrogen), along with $1 \mu \mathrm{M}$ of a live cell probe, Calcein AM (Invitrogen). Confocal images taken directly of DHE and Calcein AM stained chondrocytes of osteochondral explants. Viability of $Z$-series confocal images of Calcein AM is green staining of the cytoplasm in stained chondrocytes. Five sites explant were imaged on a BioRad 1024 Confocal Microscope equipped with a krypton/ argon laser in a custom-designed stage for live cell imaging (Central Microscopy Research Facility, University of Iowa). The sites were scanned to a depth of $150 \mu \mathrm{m}$ at $20 \mu \mathrm{m}$ intervals. Laser power was set at $10 \%$ and the iris was set at 3.5 during imaging. The exposure time was $11.5 \mathrm{~s}$ for per frame. The excitation wavelengths were $568 \mathrm{~nm}$ and $488 \mathrm{~nm}$ for scanning. Imaging sites were selected at random but care was taken to identify the sites close to the radial center of the indented region in explants. $20 \times$ image stacks were acquired and utilized for chondrocyte shape analysis [13-16]. 


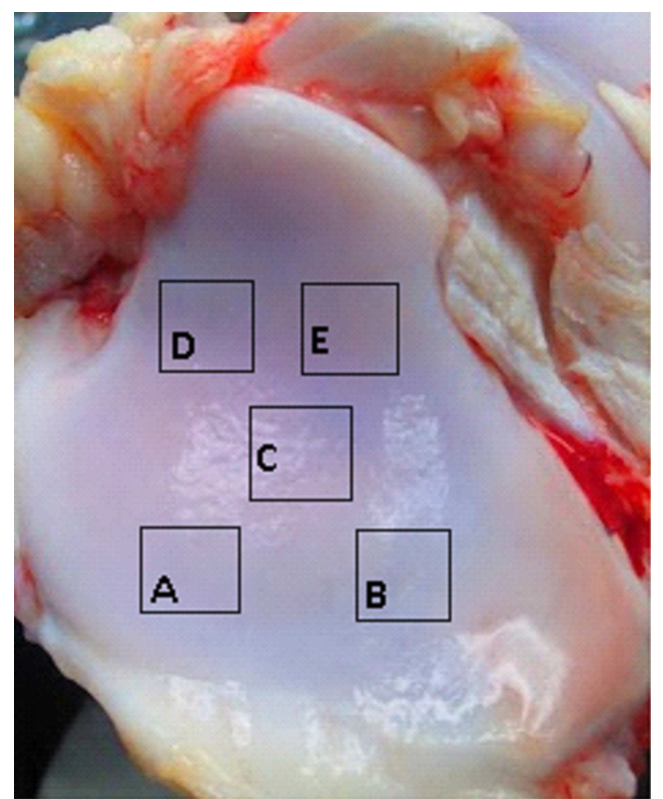

Fig. 1. Five different regions of the lateral tibial plateau.

Image analysis. The ImageJ version 1.41 was used to analyze live cells from confocal images. We calculated the area of the cell, the long (major) and short (minor) axes inside the cell, the cell angle, the circularity of the cell, and the aspect ratio. The cell coordinates were also measured. ImageJ version 1.41 for Mac OS X was obtained from the NIH website (ImageJ, Rasband, W.S., U.S. National Institutes of Health, Bethesda, MD, http://rsb.info.nih.gov/ij/, 1997-2008).

\section{Results and discussion}

In this study, we studied chondrocyte shapes and angles of bovine articular cartilage from lateral tibial plateau using confocal microscopy imaging methods.

As seen in Fig. 2c, the chondrocytes in $\mathrm{C}$ region are more deformed than in other regions.

The lengths of major and minor axes of the five different regions are given in Figs. 3 and 4 .

The loads were estimated according to the position of the regions. Since regions $A$ and $B$ of the lateral tibial plateau are at almost the same angle, it is estimated that the loads are similar in these regions. Previous research $[17,18]$ has found when the tissue was loaded, the surface cells were compressed, squeezing them into an oval shape and increasing the aspect ratio of the cells. When the load increases, the cell length increases. The research of the current study supports those previous studies [17, 18].

According to their relative positions, the loads on the regions $A$ and $B$ of the lateral tibial plateau are of similar level. The load on the regions $D$ and $E$ are similarly estimated to be almost equal, while the load on region $C$ is estimated to be in between.
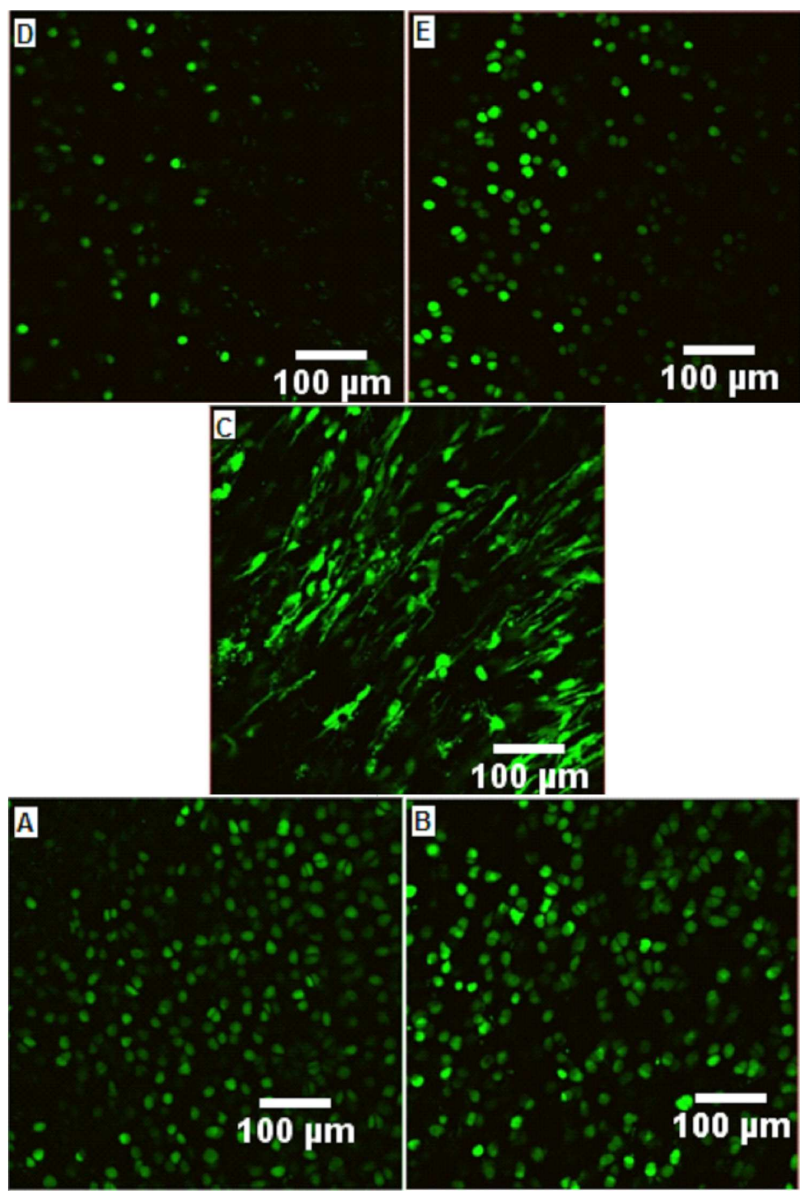

Fig. 2. Confocal images for different regions $(540 \mu \mathrm{m}$ each side).

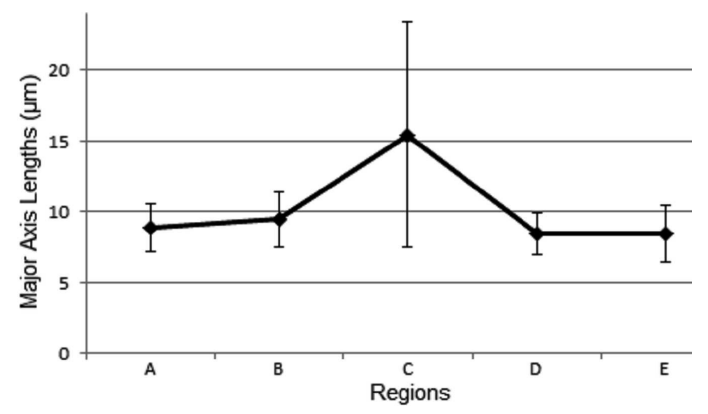

Fig. 3. The lengths of the long (major) axes of chondrocytes for different regions (cell numbers $A$ : 565, $B: 305, C: 616, D: 242, E: 462)$.

According to analyzed live cells from confocal images, the length of the long (major) axis of the region $C$ $(15.43 \pm 7.92 \mu \mathrm{m})$ is greater than the lengths of the long axes of the other regions (Fig. 3). The long axis lengths of the regions $A$ and $B$ are similar $(8.86 \pm 1.73 \mu \mathrm{m}$ and $9.46 \pm 1.97 \mu \mathrm{m}$, respectively), although greater than that of the regions $D$ and $E$, which are almost equal to each other $(8.43 \pm 1.51 \mu \mathrm{m}$ and $8.46 \pm 1.98 \mu \mathrm{m}$, respectively). 


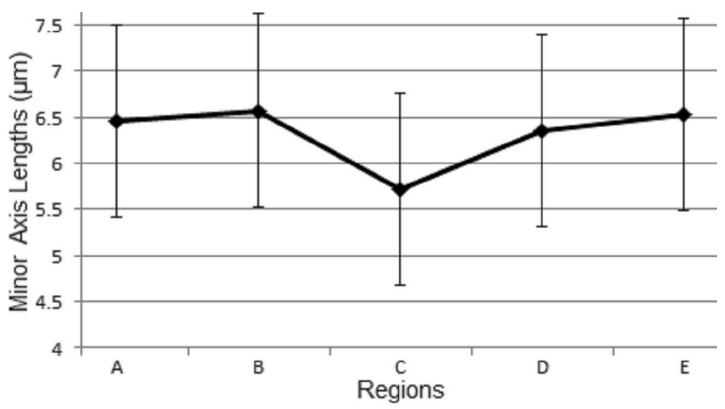

Fig. 4. The lengths of the short (minor) axes of chondrocytes for different regions (cell numbers $A$ : 565, $B: 305, C: 616, D: 242, E: 462)$.

On the other hand, the length of the short (minor) axis of the region $C$ is smaller than the lengths of the short axes of the other regions. The other axis lengths are ordered as follows: $B>E>A>D$. Since the loads on $D$ and $E$ are smaller than the loads on the other regions, the lengths of the long axes of $D$ and $E$ are found to be smaller than all the other lengths. However, although the loads on $A$ and $B$ are greater than the others, the lengths of the long axis of region $C$ is greater than that of $A$ and $B$. Similarly, even though the loads on $A$ and $B$ are greater than the loads on the other regions, the length of the short axis of $C$ is the smallest of all short axis lengths. We observed that the aspect ratios are ordered as follows: $C>B>A>D>E$.

Let us note also that the circularity of $C$ is the smallest (Table). The precision of the length was $0.01 \mu \mathrm{m}$ (Table). One-way analysis of variance (ANOVA) was used for statistical analysis. A $p$-value less than 0.05 was considered significant. For the all parameters, $p<0.01$ was observed between the groups. Only differences between the measured angles was insignificant $(p=0.307)$. This analysis explains why the regions $A$ and $B$ (in Fig. 1) have less natural loading than region $C$. Regions $A$ and $B$ appear to be covered by a meniscus, which is responsible for shock absorption, load transmission, and stability within the knee joint [15].

Parameters describing chondrocytes' morphology determined from ImageJ analysis.

TABLE

\begin{tabular}{c|c|c|c|c|c|c}
\hline \hline Region & Area $\left[\mu \mathrm{m}^{2}\right]$ & $\begin{array}{c}\text { Major axis } \\
\text { lengths }[\mu \mathrm{m}]\end{array}$ & $\begin{array}{c}\text { Minor axis } \\
\text { lengths }[\mu \mathrm{m}]\end{array}$ & Angle $\left[{ }^{\circ}\right]$ & Circularity & Aspect ratio \\
\hline$A$ & $45.37 \pm 14.02$ & $8.86 \pm 1.73$ & $6.45 \pm 1.04$ & $94.89 \pm 44.70$ & $0.54 \pm 0.19$ & 1.40 \\
$B$ & $49.53 \pm 17.18$ & $9.46 \pm 1.97$ & $6.57 \pm 1.04$ & $75.94 \pm 40.88$ & $0.64 \pm 0.18$ & 1.47 \\
$C$ & $71.74 \pm 60.82$ & $15.43 \pm 7.92$ & $5.72 \pm 1.04$ & $57.74 \pm 37.46$ & $0.42 \pm 0.21$ & 3.05 \\
$D$ & $42.67 \pm 13.87$ & $8.43 \pm 1.51$ & $6.35 \pm 1.04$ & $86.09 \pm 62.41$ & $0.79 \pm 0.12$ & 1.34 \\
$E$ & $44.16 \pm 15.45$ & $8.46 \pm 1.98$ & $6.53 \pm 1.04$ & $107.42 \pm 53.33$ & $0.82 \pm 0.13$ & 1.30 \\
$P$ value & $p<0.01$ & $p<0.01$ & $p<0.01$ & $p=0.307$ & $p<0.01$ & $p<0.01$
\end{tabular}

Articular cartilage is a highly organized avascular tissue acting as a shock absorber that dampens physiologic loads, and transfers the applied load to the subchondral bone [3]. Chondrocytes form a sparse cell population and are responsible for the secretion of extracellular matrix components of cartilage such as proteoglycans and collagen type II fibrils, the three-dimensional meshwork of which are directly relevant to the load carriage functioning of a particular articular cartilage region. Previous in vivo [19] and in vitro [20] studies have shown that mechanical loading modulates the biosynthetic response of chondrocytes in articular cartilage explants [7]. Our results suggest that the mechanical loading affects also the tibial plateau cartilage morphology (angles, long and short axes, circularity and area of the chondrocytes).

Quantitative studies on the effects of static loading on cartilage volume and thickness have proposed that structural changes in the cartilage matrix occur with physiological loading [15, 21, 22]. The natural load effects on the different morphological parameters of chondrocytes from different regions of articular cartilage of the tibial plateau, however, have not been studied before using confocal microscopy imaging techniques. The acute response effects of load experiments on healthy cartilage, which has been the subject of many previous studies [15, 21-24], may be insufficient to understand physiological loading due to lack of information about accessory mechanisms, the relative responses in positional different regions of the cartilage and the deformations through natural aging process. Our study contributes to the understanding of such phenomena.

\section{Conclusions}

In this paper we have shown the natural load effects on the different morphological parameters of chondrocytes. The more loaded regions have smoother chondrocytes comes out from our results.

Further studies are necessary to determine differences among chondrocyte shapes and collagen fibrils orientation in different regions of the cartilage. Investigation of 
mechanisms of load carriage in cartilage may play important roles in the development of new therapies for osteoarthritis.

\section{References}

[1] T. Nguyen, A. Oloyede, Proc. Inst. Mech. Eng. H 215, 565 (2001).

[2] A. Oloyede, N.D. Broom, Clin. Biomech. 9, 149 (1994).

[3] T. Nagai, K.S. Furukawa, M. Sato, T. Ushida, J. Mochida, Tissue Eng. Part A 14, 1183 (2008).

[4] J.A. Buckwalter, H.J. Mankin, J. Bone Joint Surg. Am. 79, 612 (1997).

[5] E.B. Hunziker, Osteoarthr. Cartil. 10, 432 (2001).

[6] F. Guilak, D.L. Butler, S.A. Goldstein, Clin. Orthop. 391, 295 (2001).

[7] N.O. Chahine, C.T. Hung, G.A. Ateshian, Eur. Cell Mater. 13, 100 (2007).

[8] C.P. Neu, A. Khalafi, K. Komvopoulos, T.M. Schmid, A.H. Reddi, Arthritis Rheum. 56, 3706 (2007).

[9] G.E. Nugent, N.M. Aneloski, T.A. Schmidt, B.L. Schumacher, M.S. Voegtline, R.L. Sah, Arthritis Rheum. 54, 1888 (2006).

[10] K. Subburaj, A. Valentinitsch, A.B. Dillon, G.B. Joseph, X. Li, T.M. Link, T.P. Vail, S. Majumdar, Magn. Reson. Imaging 31, 1129 (2013).

[11] B. Bittersohl, F.R. Miese, H.S. Hosalkar, M. Herten, G. Antoch, R. Krauspe, C. Zilkens, Osteoarthr. Cartil. 20,653 (2012).

[12] T. Xie, Y. Xia, S. Guo, P. Hoover, Z. Chen, Z, G.M. Peavy, J. Biomed. Opt. 13, 054034 (2008).
[13] K.J. Wolff, P.S. Ramakrishnan, M.J. Brouillette, B.J. Journot, T.O. McKinley, J.A. Buckwalter, J.A. Martin, J. Orthop. Res. 31, 191 (2013).

[14] P.S. Ramakrishnan, D.R. Pedersen, N.J. Stroud, D.J. McCabe, J.A. Martin, Proc. Inst. Mech. Eng. H 225, 993 (2011).

[15] K. Subburaj, D. Kumar, R.B. Souza, H. Alizai, X. Li, T.M. Link, S. Majumdar, Am. J. Sports Med. Sep. 40, 2134 (2012).

[16] W. Goodwin, D. McCabe, E. Sauter, E. Reese, M. Walter, J.A. Buckwalter, J.A. Martin, J. Orthop. Res. 28, 1057 (2010).

[17] H.A. Alhadlaq, Y. Xia, F.M. Hansen, C.M. Les, G. Lust, Connect. Tissue Res. 48, 76 (2007).

[18] E. Cicek, F. Badar, J. Li, X. Tian, Y. Xia, in: Proc. 4 th Int. Conf. Bioinformatics Biomedical Engineering, Chengdu, China, Ed.: K.-C. Chou, IEEE, Piscataway 2010 , p. 1.

[19] L.A. Setton, V.C. Mow, D.S. Howell, J. Orthop. Res 13, 473 (1995).

[20] M. Wong, M. Siegrist, X. Cao, Matrix Biol. 18, 391 (1999).

[21] C. Liess, S. Lusse, N. Karger, M. Heller, C.C. Gluer, Osteoarthr. Cartilage 10, 907 (2002).

[22] M.J. Kaab, K. Ito, J.M. Clark, H.P. Notzli, J. Orthop. Res. 16, 743 (1998).

[23] Y. Xia, H. Alhadlaq, N. Ramakrishnan, A. Bidthanapally, F. Badar, M. Lu, J. Struct. Biol. 164, 88 (2008).

[24] H. Muir, BioEssays 17, 1039 (1995). 